Open Access

\title{
nCounter PanCancer Immune Profiling Panel (NanoString Technologies, Inc., Seattle, WA)
}

\author{
Alessandra Cesano
}

The $n$ Counter PanCancer Immune Profiling Panel is a unique 770-plex gene expression panel to measure the human immune response in both solid and liquid cancer types. The panel measures many features of the immune response to facilitate rapid development of clinical actionable gene expression profiles in the context of cancer immunotherapy. The assay is run on the $n$ Counter Analysis System (Nanostring Technologies, Inc.), an automated system which received $510(\mathrm{k})$ clearance from the FDA for use with the Prosigna Breast Cancer Prognostic Gene Signature Assay [1]. The $n$ Counter Analysis System is based on a novel digital color-coded barcode technology which allows for direct multiplexed measurement of gene expression from low amount of mRNA (25 to $300 \mathrm{ng}$ ) without need for amplification [2]. The technology uses molecular "barcodes" and single molecule imaging to detect and count (completely digital) hundreds of unique transcripts in a single reaction with high precision and sensitivity $(<1$ copy per cell). Each color-coded optical barcode is attached to a single target-specific hybridization probe corresponding to a gene of interest. Mixed together with controls, they form multiplexed "CodeSets" which are provided as ready to use reagents (Fig. 1). Currently three "CodeSets" are available as ready to use reagents for oncology investigations: a) the PanCancer Pathways - a panel of 700 essential genes representing major cancer pathways including key driver genes b) the PanCancer progression - a panel of 770 genes from 4 major biologic processes that contribute to increased tumor growth and invasiveness including angiogenesis, epithelial to mesenchymal transition and extra-matrix remodeling and metastasis and c) the PanCancer Immune profiling panel (described in more details below). Multiple CodeSets can be run on the same samples thus proving integrated information about both tumor and host immune response.
At a very high level, the assay includes three main steps:

1. Hybridization: unique pairs of a "capture" and a "reporter" probe are provided for each gene of interest, allowing up to 800 genes to be multiplexed, and their mRNA transcript levels measured, in a single experiment, for each sample. The "reporter" probe carries the signal, and the "capture" probe allows the complex to be immobilized for data collection.

2. Purification and immobilization: after hybridization, samples are transferred to the nCounter Prep Station where excess probes are removed and probe/ target complexes are bound, immobilized, and aligned on the $n$ Counter Cartridge.

3. Counting and Analysis: sample cartridges are placed in the $n$ Counter Digital Analyzer for data collection.

The time from sample lysates to data results is two days and because the process is highly automated the hands-on time (and therefore room for human errors) is limited (25 min per 12 samples). Measurements are performed using the commercially available nCounter Analysis Instrumentation at the site of sample collection or through working with any of the multiple Contract Research Organizations offering NanoString services.

For application of this technology to immune-oncology the $n$ Counter PanCancer Immune Profiling Panel provides a highly multiplexed gene expression panel designed to quantitate 770 genes that fall into four functional categories (Fig. 1):

1. Identifying 24 different infiltrating immune cell types, such as those in a peripheral blood mononuclear cells (PBMC) population or infiltrating into a tumor.

Correspondence: acesano@nanostring.com

NanoString Technologies, Inc., Seattle, WA, USA 


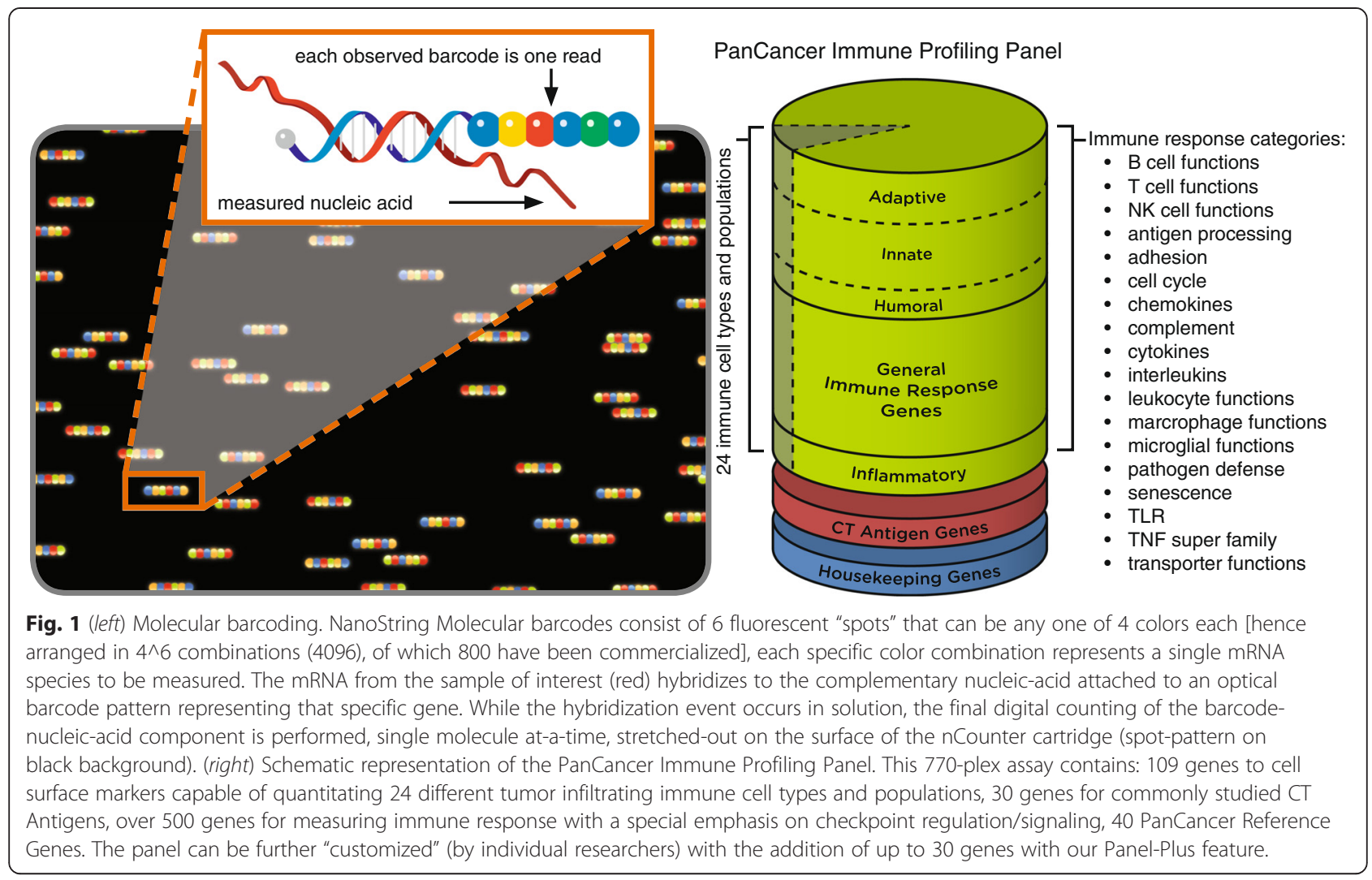

2. Assessing immunological function and response to immunotherapy, such as immune checkpoint regulation.

3. Identifying tumor-specific antigens, such as cancertestis (CT) antigens.

4. Housekeeping genes that facilitate sample-to-sample normalization.

\section{Type of data obtained/readout}

Barcodes are counted and tabulated for each target by the $n$ Counter Digital Analyzer. The data readouts are either the composite of the individual expression profile of cells within that population; or detailed, single-cell level expression, which may represent biologically relevant small percentage $(5-10 \%)$ of the entire population of the cells. The instrument analysis software automatically performs QC, normalization, data analysis and creates multi-page reports with the options of performing advanced analyses including pathway applications.

\section{Limitations of the approach}

- The data are not spatially resolved, hence, it represents the average of a few 1000's of cells

- The data are targeted discovery (as contrasted with pure discovery), and measure only the 770 genes predefined in the panel. It is possible to add 30 completely custom genes to the 770-plex panel (total of 800-plex)

- While it is possible to resolve alternate splice transcripts and expressed gene-fusions, the panel does not measure single-nucleotide polymorphisms (SNPs).

\section{Advantages of the approach}

- Multiplex hundreds of gene targets in a single reaction

- High sensitivity (<1 copy per cell)

- Fully-automated system

- No enzymes or amplification required to perform assay, ideally suited for FFPE samples and cell lysates

- Multiplex 800 regions from as little as 25-300 ng of total RNA

- Completely digital detection (all quantitation is by direct single-molecule counting)

- Automated analysis software

Types of samples needed and special issues pertaining to samples

The nCounter PanCancer Immune profiling panel is fully compatible with clinically relevant sample types such as fresh-frozen (FF) tissue, formalin-fixed paraffin-embedded (FFPE) tumor sections, isolated immune cell populations 
such as PBMC and cell lysates. For very low input samples (even down to single-cell work, such at CTCs), a multiplexed target enrichment amplification protocol is available.

\section{Level of evidence}

There have been bout over 800 peer-reviewed publications using the nCounter Analysis System. The instrument, reagents and software have received $510(\mathrm{k})$ clearance from the FDA for use with the Prosigna Breast Cancer Prognostic Gene Signature Assay [1]. There is a growing body of literature available demonstrating the use of the $n$ Counter analysis system using much of the same content as in the $n$ Counter PanCancer Immune Profiling Panel in immunooncology setting [3].

\section{Abbreviations}

CT: Cancer testis antigens; CTC: Circulating tumor cells; FF: Fresh-frozen; FFPE: Formalin-fixed paraffin-embedded; FDA: Food and Drug

Administration; PBMC: Peripheral blood mononuclear cells; QC: Quality check; RNA: Ribonucleic acid; SNPs: Single-nucleotide polymorphisms.

\section{Competing interest}

Alessandra Cesano MD, PhD is employed by the NanoString Technologies, Inc., Seattle, WA.

Received: 11 August 2015 Accepted: 15 August 2015

Published online: 15 December 2015

\section{References}

1. Nielsen T, Wallden B, Schaper C, Ferree S, Liu S, Gao D, et al. Analytical validation of the PAM50-based Prosigna Breast Cancer Prognostic Gene Signature Assay and nCounter Analysis System using Formalin-fixed paraffin-embedded breast tumor specimens. BMC Cancer. 2014;14:177-204.

2. Geiss GK, Bumgarner RE, Birditt B, Dahl T, Dowidar N, Dunaway DL, et al. Direct multiplexed measurement of gene expression with color-coded probe pairs. Nat Biotechnol. 2008;26:317-25.

3. Liu L, Mayes PA, Eastman S, Shi H, Yadavilli S, Zhang T, et al. The BRAF and MEK Inhibitors Dabrafenib and Trametinib: Effects on Immune Function and in Combination with Immunomodulatory Antibodies Targeting PD1, PD-L1 and CTLA-4. Clin Cancer Res. 2015;21(7):1639-51.

\section{Submit your next manuscript to BioMed Central and take full advantage of:}

- Convenient online submission

- Thorough peer review

- No space constraints or color figure charges

- Immediate publication on acceptance

- Inclusion in PubMed, CAS, Scopus and Google Scholar

- Research which is freely available for redistribution 